\title{
Chemical structures of hydrazine-treated graphene oxide and generation of aromatic nitrogen doping
}

\author{
Sungjin Park', Yichen Hu², Jin Ok Hwang ${ }^{3}$, Eui-Sup Lee ${ }^{4}$, Leah B. Casabianca², Weiwei Cai ${ }^{5}$, Jeffrey R. Potts ${ }^{5}$, \\ Hyung-Wook Ha ${ }^{5}$, Shanshan Chen ${ }^{5}$, Junghoon Oh${ }^{1}$, Sang Ouk Kim ${ }^{3}$, Yong-Hyun Kim ${ }^{4}$, Yoshitaka Ishii ${ }^{2,6}$ \\ \& Rodney S. Ruoff 5
}

Chemically modified graphene platelets, produced via graphene oxide, show great promise in a variety of applications due to their electrical, thermal, barrier and mechanical properties. Understanding the chemical structures of chemically modified graphene platelets will aid in the understanding of their physical properties and facilitate development of chemically modified graphene platelet chemistry. Here we use ${ }^{13} \mathrm{C}$ and ${ }^{15} \mathrm{~N}$ solid-state nuclear magnetic resonance spectroscopy and X-ray photoelectron spectroscopy to study the chemical structure of ${ }^{15} \mathrm{~N}$ labelled hydrazine-treated ${ }^{13} \mathrm{C}$-labelled graphite oxide and unlabelled hydrazine-treated graphene oxide, respectively. These experiments suggest that hydrazine treatment of graphene oxide causes insertion of an aromatic $\mathrm{N}_{2}$ moiety in a five-membered ring at the platelet edges and also restores graphitic networks on the basal planes. Furthermore, density-functional theory calculations support the formation of such $\mathrm{N}_{2}$ structures at the edges and help to elucidate the influence of the aromatic $\mathrm{N}_{2}$ moieties on the electronic structure of chemically modified graphene platelets.

\footnotetext{
${ }^{1}$ Department of Chemistry, Inha University, Incheon 402-751, Korea. ${ }^{2}$ Department of Chemistry, University of Illinois at Chicago, 845 W Taylor Street, Chicago, Illinois 60607, USA. ${ }^{3}$ Department of Materials Science and Engineering, KAIST, Daejeon 305-701, Korea. ${ }^{4}$ Graduate School of Nanoscience and Technology (WCU) and KAIST Institute for the NanoCentury, KAIST, Daejeon 305-701, Korea. ${ }^{5}$ Department of Mechanical Engineering and the Materials Science and Engineering Program, University of Texas at Austin, One University Station C2200, Austin, Texas 78712-0292, USA. ${ }^{6}$ Center for Structural Biology, University of Illinois at Chicago, 1100 South Ashland Street, Chicago, Illinois 60607, USA. Correspondence and requests for materials should be addressed to S.P. (email: sungjinpark@inha.ac.kr).
} 
$\mathrm{n}$ the 1960 s, hydrazine $\left(\mathrm{NH}_{2} \mathrm{NH}_{2}\right)$ was used to chemically reduce thin graphite oxide (GO) particles ${ }^{1,2}$; Ruoff and co-workers ${ }^{3}$ developed the use of hydrazine to reduce individual graphene oxide platelets four decades later. For the last half decade, among several chemical reductants ${ }^{4,5}$, hydrazine has been the most commonly used reductant due to its ease of use (for example, via a one-pot synthesis in either liquid or gas phase) and its ability to achieve a high degree of reduction of graphene oxide without the need for further treatment ${ }^{3,6-8}$. Hydrazine-treated graphene oxide (chemically reduced graphene oxide, 'CReGO') ${ }^{3}$ is one of the most promising graphene-based materials for several applications, such as polymer composites, ultracapacitors, rechargeable batteries, chemical/biosensors and thin films ${ }^{9-14}$. A large fraction of the oxygen-based functional groups of graphene oxide are removed by exposure to hydrazine; however, the resulting graphene materials contain a small amount of $\mathrm{O}$ and $\mathrm{N}$ atoms (with approximate $\mathrm{C} / \mathrm{O}$ and $\mathrm{C} / \mathrm{N}$ ratios of 10 and 22 , respectively) ${ }^{3,6}$. The presence of these heteroatoms on the 'graphene' platelets-namely, electron donating $\mathrm{N}$ atoms and electron withdrawing $\mathrm{O}$ atoms - may create a doping effect that considerably affects their electronic structure ${ }^{15}$. Also, chemical moieties containing heteroatoms or heterocycles can be utilized to open a new path to selective chemical modifications of graphene-based systems. However, despite the use of CReGO in a variety of different ways ${ }^{9-11}$, the chemical identities of the functional groups containing those heteroatoms have not yet been fully determined.

${ }^{13} \mathrm{C}$ solid-state nuclear magnetic resonance (SSNMR) spectroscopy may be the most powerful method to study the detailed chemical structures of graphene-based materials such as graphene oxide, GO, reduced graphene oxide and other chemically modified graphenes $(\mathrm{CMGs})^{16,17}$. However, the low natural abundance $(1.1 \%)$ of the ${ }^{13} \mathrm{C}$ isotope results in a low signal-to-noise ratio, which, along with the broad peaks typically observed from CMGs, precludes the use of multidimensional SSNMR. This was the motivation for our recent work, which used $99 \%{ }^{13} \mathrm{C}$-labelled graphite made by chemical vapour deposition (CVD) as the precursor to ${ }^{13} \mathrm{C}$-labelled GO, which was studied using ${ }^{13} \mathrm{CSSNMR}^{18,19}$. This investigation revealed that hydroxyl, 1,2-epoxide and $\mathrm{sp}^{2}$ carbons on the basal planes ${ }^{20}$ are directly bonded to each other in GO. It has been proposed that $\mathrm{sp}^{2}$ networks in GO are terminated with carboxyl and ketone groups, predominately at the edges of the structure ${ }^{21}$. Separately, the existence of five- or six-membered lactol rings at the edges of GO has been suggested by Gao et $a^{22}$. While considerable structural information has been obtained for GO, few structural details, especially at the platelet edges, have been obtained for CReGO or unexfoliated GO reduced by hydrazine, the latter of which we define as reduced graphite oxide or 'RGO'.

For SSNMR measurements, a protocol to produce ${ }^{13} \mathrm{C}$ - and ${ }^{15} \mathrm{~N}$ labelled RGO by reducing ${ }^{13} \mathrm{C}$-labelled GO with ${ }^{15} \mathrm{~N}$-hydrazine monohydrate was developed, and ${ }^{1} \mathrm{H} /{ }^{13} \mathrm{C} /{ }^{15} \mathrm{~N}$ triple-resonance SSNMR experiments were performed on the samples. SSNMR spectroscopy of $\mathrm{N}$ typically requires the use of isotopically labelled samples because of the low natural abundance of ${ }^{15} \mathrm{~N}(\sim 0.4 \%)$ and also large quadrupolar interactions of naturally abundant ${ }^{14} \mathrm{~N}$ nuclei. Herein, we present new structural insight into reduced graphene oxide materials, revealed by ${ }^{13} \mathrm{C}$ - and ${ }^{15} \mathrm{~N}$ rotational-echo doubleresonance (REDOR) SSNMR experiments ${ }^{23,24}$ on ${ }^{13} \mathrm{C}$ - and ${ }^{15} \mathrm{~N}$ labelled RGO as well as by X-ray photoelectron spectroscopy (XPS) measurements on unlabelled, hydrazine-treated graphene oxide. This work helps to provide a deeper understanding of the chemical structures and electronic properties of reduced graphene oxides.

\section{Results}

NMR study of isotope-labelled RGO samples. A ${ }^{13} \mathrm{C}$-labelled graphite film was grown by $\mathrm{CVD}$ of ${ }^{13} \mathrm{C}$-enriched methane on $\mathrm{Ni}$ foil as described previously ${ }^{18}$. Raman spectroscopy confirmed that the as-grown graphite film was of high quality and highly enriched $(99 \%)$ in the ${ }^{13} \mathrm{C}$ isotope (Supplementary Fig. S1) ${ }^{18}$. As expected, the first-order G-band was observed at $1,518 \mathrm{~cm}^{-1}$, while the D-band typically detected at $1,297 \mathrm{~cm}^{-1}$ was completely absent. This result indicates high-quality films and an enrichment of approximately $99 \%{ }^{13}$ C. ${ }^{13}$ C-labelled graphite film was removed from the $\mathrm{Ni}$ substrate by scotch tape, then rinsed and sonicated in acetone. As shown in Supplementary Figure S1, the graphite film was cut into small pieces $(10-50 \mu \mathrm{m})$. This ${ }^{13} \mathrm{C}$-labelled graphite was then oxidized to ${ }^{13} \mathrm{C}$-labelled $\mathrm{GO}$, and subsequently reduced using $98 \%$ ${ }^{15} \mathrm{~N}$-labelled hydrazine monohydrate to produce ${ }^{13} \mathrm{C}$-, ${ }^{15} \mathrm{~N}$-labelled RGO (see the Methods section for experimental details).

Figure 1 shows high-resolution magic angle spinning (MAS) ${ }^{13} \mathrm{C}$-SSNMR spectra of (a) the labelled GO and (b) the labelled RGO
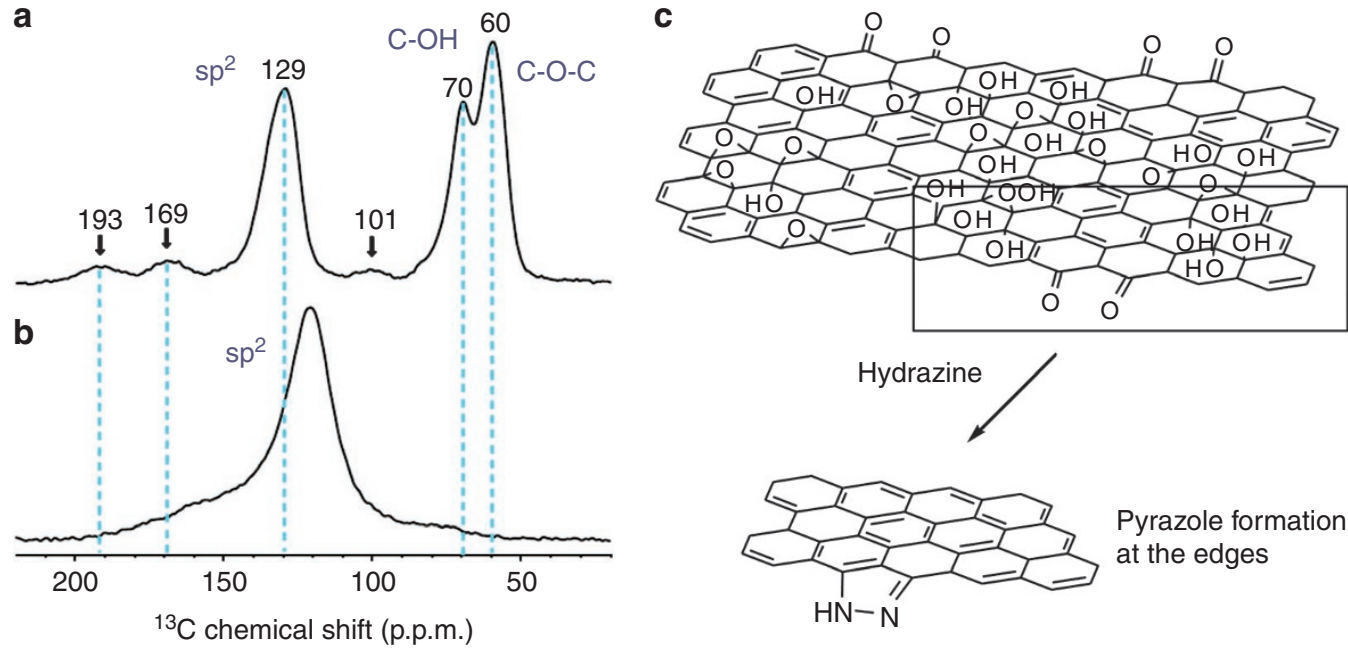

Figure 1 | Chemical structures of labelled samples before and after hydrazine treatment. ${ }^{13} \mathrm{C}$ MAS SSNMR spectra of (a) ${ }^{13} \mathrm{C}-$ labelled GO and (b) ${ }^{13} \mathrm{C}$ - and ${ }^{15} \mathrm{~N}$-labelled RGO. The spectrum in a was reproduced from Figure 1a in our previous work by Cai et al. ${ }^{18}$ Reprinted with permission from AAAS. All the spectra were obtained with direct ${ }^{13} \mathrm{C}$ excitation by a $\pi / 2$ pulse at a ${ }^{13} \mathrm{C}$ NMR frequency of $100.643 \mathrm{MHz}$ and with $20 \mathrm{kHz} \mathrm{MAS}$. (c) A schematic illustrating pyrazole formation at the edges of the platelets during hydrazine treatment of graphene oxide $(-\mathrm{COOH}$ groups at the platelet edges have been omitted for clarity). 
samples. High-resolution SSNMR spectroscopy using MAS has been used to study the chemical structures of $\mathrm{GO}^{3,18,22}$. A spectrum of the labelled RGO sample (Fig. 1b) shows an excellent signal-tonoise ratio, despite the small sample quantities of approximately $2 \mathrm{mg}$ and a relatively short acquisition time of $21 \mathrm{~min}$. Notably, obtaining a spectrum of similar quality from an unlabelled sample would require signal accumulation for nearly 140 days. There are several obvious spectral changes from the samples before and after hydrazine treatment. In Figure 1a, there is a weaker peak at $193 \mathrm{p}$. p.m., which was previously assigned to ketone groups $\left(\mathrm{RR}^{\prime} \mathrm{C}=\mathrm{O}\right)$ at the edges of the platelets ${ }^{22}$. The edge structures could be present around hole defects or torn edges in the interior of the platelets ${ }^{25,26}$. The high sensitivity resulting from our novel isotope labelling method is especially useful for SSNMR analysis of important species with lower populations. Interestingly, the peak at 193 p.p.m. disappeared in Figure 1b, suggesting that the changes occurred in the chemical structure at the edges. As GO is thermally unstable, thermal treatment of GO under our reaction conditions might lead to changes in the chemical structure of GO. However, stirring GO in water at $80^{\circ} \mathrm{C}$ without hydrazine resulted in little change of the chemical structure of GO, as shown in Supplementary Figure S2. These results confirm that hydrazine is responsible for the changes in the chemical structure of graphene oxide.

Advanced SSNMR experiments correlating the connectivity of ${ }^{13} \mathrm{C}$ and ${ }^{15} \mathrm{~N}$ have been used for ${ }^{13} \mathrm{C}$ - and ${ }^{15} \mathrm{~N}$-labelled biomolecules $^{27-29}$; however, for graphene-based materials, such SSNMR experiments have not been performed to date, partly because of the difficulties in the preparation of appropriate samples. In this work, we acquired the ${ }^{13} \mathrm{C}$ MAS SSNMR spectra without and with ${ }^{13} \mathrm{C}-$ ${ }^{15} \mathrm{~N}$ dipolar dephasing using a REDOR pulse sequence, as shown in Figure 2a,b, respectively. Details on the pulse sequence are provided in Figure $2 \mathrm{~d}$ (refs 23,24 ). The ${ }^{13} \mathrm{C}$ atoms associated with ${ }^{15} \mathrm{~N}$ atoms lose their signal in the ${ }^{13} \mathrm{C}$ MAS spectrum with ${ }^{13} \mathrm{C}-{ }^{15} \mathrm{~N}$ dephasing. Subtraction of Figure $2 \mathrm{~b}$ from Figure $2 \mathrm{a}$ shows NMR signals of the ${ }^{13} \mathrm{C}$ atoms that are one or at most two bonds away from ${ }^{15} \mathrm{~N}$ atoms ${ }^{24}$. The resulting difference spectrum, shown in Figure 2c, reveals a new spectral feature around 150 p.p.m. (labelled with an arrow in Fig. 2c), which has not been reported previously. The broad signal with two maxima at 140 and 157 p.p.m. cannot be observed as a separate peak in the spectrum shown in Figure 2 a without ${ }^{13} \mathrm{C}-$ ${ }^{15} \mathrm{~N}$ dephasing, as they are overlapped with a major peak centred at 118 p.p.m. In an attempt to eliminate the effect of any associated ${ }^{15} \mathrm{~N}$-labelled contaminants resulting from the ${ }^{15} \mathrm{~N}$-labelled hydrazine treatment, the RGO sample was thoroughly washed with acetone and subsequently dried under high vacuum; then the REDOR experiment was repeated. The REDOR experiment on the washed RGO sample yielded an almost identical result to that shown in Figure 2 (data not shown). Because the natural abundance of ${ }^{15} \mathrm{~N}$ is extremely low, this observation suggests that the RGO contains ${ }^{15} \mathrm{~N}$ species that originate from ${ }^{15} \mathrm{~N}$-labelled hydrazine and that the ${ }^{15} \mathrm{~N}$ species are likely to be chemically bonded to ${ }^{13} \mathrm{C}$ species of RGO. We found this peak at approximately 150 p.p.m. for another labelled sample, which was prepared from a separate batch (Supplementary Fig. S3).

Given these observations, we suggest that the ${ }^{13} \mathrm{C}$ NMR peak around 150 p.p.m. can be attributed to pyrazole groups-aromatic five-membered rings with two adjacent $\mathrm{N}$ atoms as shown in Figure $1 \mathrm{c}$-which usually show ${ }^{13} \mathrm{C}$ NMR signals between 150 and 160 p.p.m. ${ }^{30}$ Pyrazole groups have been produced by the reaction of diketone groups with primary amine moieties ${ }^{31}$. In addition, the formation of pyrazole groups from the reaction between hydrazine (one of the primary amine moieties) and diketone groups has been well established; for example, see the reaction shown in Supplementary Figure S4 (ref. 31). Alternatively, the new ${ }^{13} \mathrm{C}$ NMR peaks around 150 p.p.m. may be also assigned to $\mathrm{C}=\mathrm{N}$ bonds. Hydrazone groups, which contain non-aromatic $\mathrm{C}=\mathrm{N}$ double bonds, have been
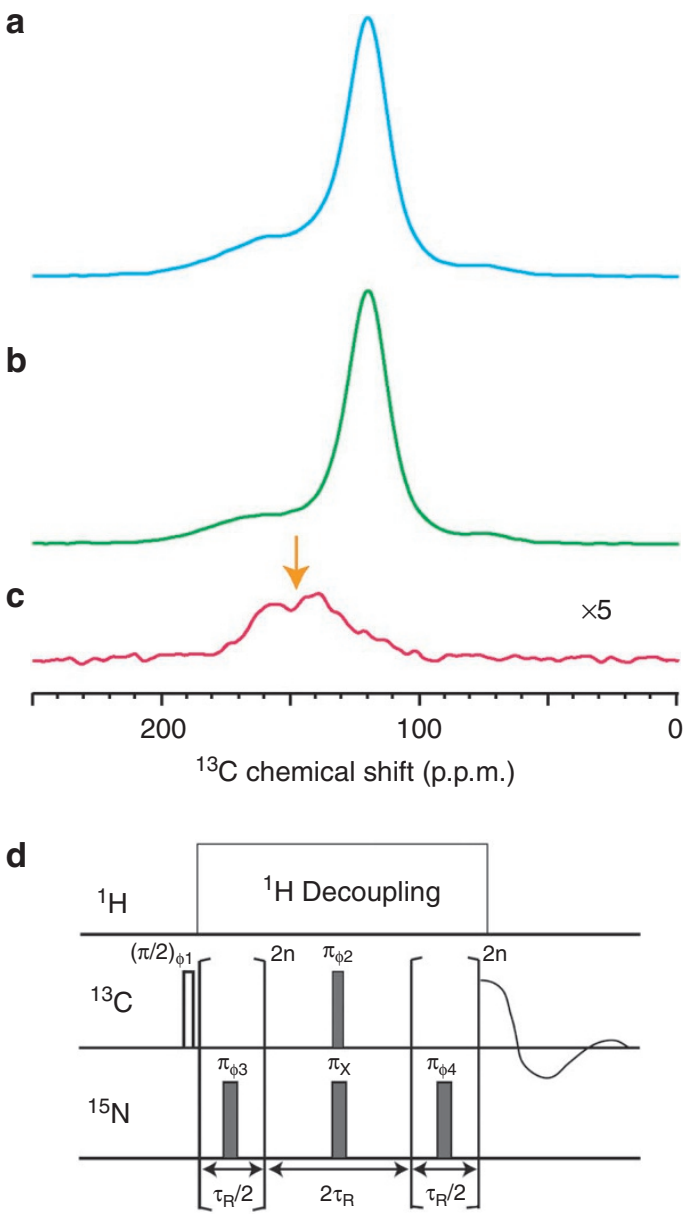

Figure $2 \mid{ }^{13} \mathrm{C}$ REDOR SSNMR measurement of labelled RGO. ${ }^{13} \mathrm{C}$ MAS SSNMR spectra with a $\pi / 2$-pulse excitation of ${ }^{13} \mathrm{C}$ - and ${ }^{15} \mathrm{~N}$-labelled RGO, (a) without and (b) with dipolar dephasing by ${ }^{13} \mathrm{C}-{ }^{15} \mathrm{~N}$ REDOR during a period of $1.6 \mathrm{~ms}$. (c) The difference spectrum of $\mathbf{a}$ and $\mathbf{b}$. Note that the vertical scale in $\mathbf{c}$ is magnified five times, compared with that of $\mathbf{a}$ and b. The spectra in $\mathbf{a}$ and $\mathbf{b}$ were collected with the pulse sequence shown (d), (a) without and (b) with ${ }^{15} \mathrm{~N} \pi$-pulse trains. Although the ${ }^{13} \mathrm{C}$ signal intensities for both control and dephased spectra are mildly modulated by ${ }^{13} \mathrm{C} \mathrm{T}_{2}$ relaxation and ${ }^{13} \mathrm{C}-{ }^{13} \mathrm{C} \mathrm{J}$-coupling, the difference spectrum in c highlights a spectrum of ${ }^{13} \mathrm{C}$ species within a few bonds from ${ }^{15} \mathrm{~N}$ atoms. The phase cycles in $\mathbf{d}$ are as follows: $\phi_{1}=x,-x, y,-y ; \phi_{2}=y, y,-x,-x$. The receiver phase was cycled as $x,-x, y,-y$. The phases for $\phi_{3}$ and $\phi_{4}$ in the rotor-synchronous $\pi$-pulse trains were incremented after every $\pi$-pulse, following the $X Y-16$ phase cycle $(x, y, x, y, y, x, y, x,-x,-y,-x,-y,-y$, $-x,-y,-x) . \tau_{R}$ denotes one rotation period of magic angle spinning.

produced by the reaction between hydrazines and ketones or aldehydes as shown in Supplementary Figure S4 (refs 32, 33). In either case, the reactions may take place at the ketone groups of GO via nucleophilic attack by the hydrazine molecules. Consequently, both reactions can explain the complete removal of the ketone peak in the ${ }^{13} \mathrm{C}$ NMR spectrum. However, it is difficult to distinguish between pyrazole and hydrazone groups based on the chemical shifts in the ${ }^{13} \mathrm{C}$ NMR spectrum.

The ${ }^{15} \mathrm{~N}$ MAS SSNMR spectrum of the ${ }^{13} \mathrm{C}$ - and ${ }^{15} \mathrm{~N}$-labelled material was obtained with cross-polarization (CP) from ${ }^{1} \mathrm{H}$ spins and is shown in Figure 3a. To the best of our knowledge, this is the first report of a ${ }^{15} \mathrm{~N}$ NMR study on a graphene-based material. The spectrum in Figure 3a shows a broad peak centred at approximately 190 p.p.m., which can be assigned to pyrazole moieties ${ }^{34}$. Because of 

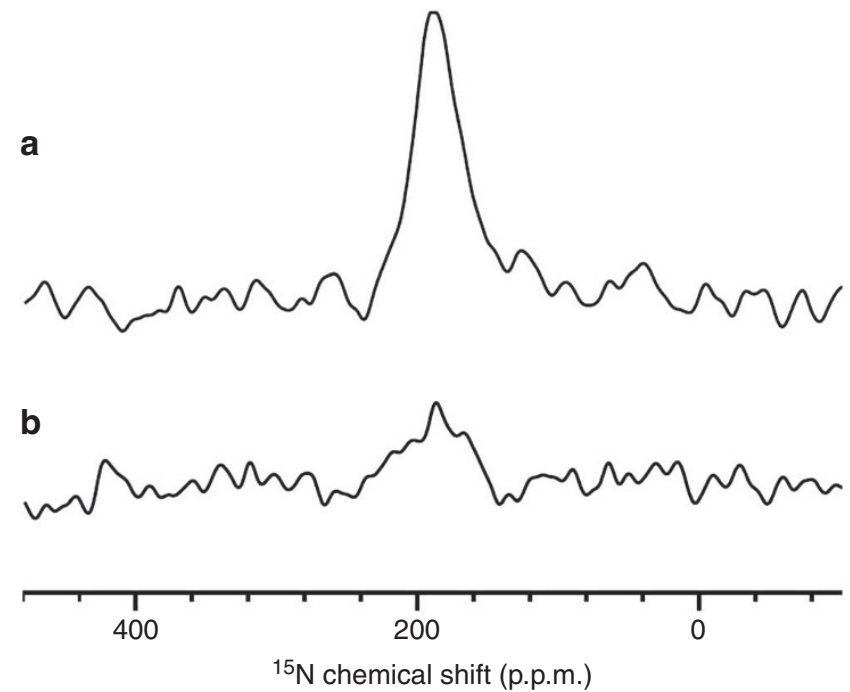

C
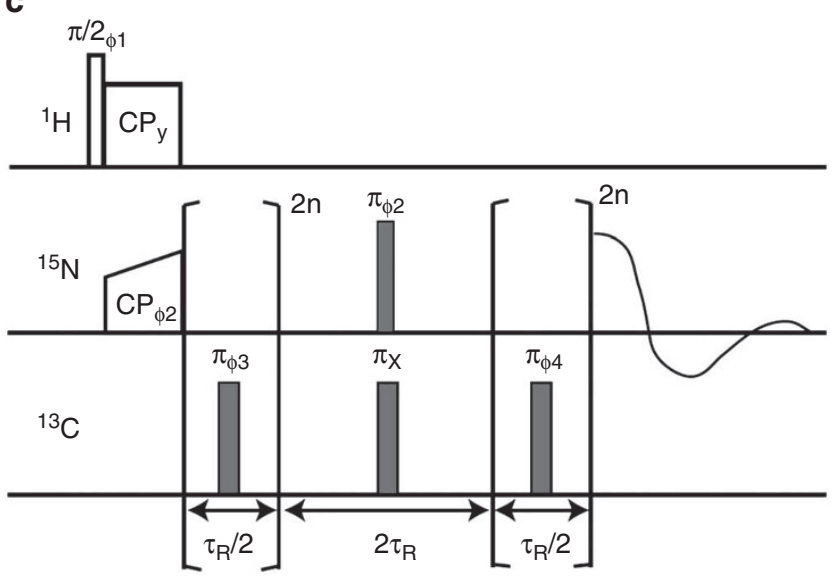

Figure 3 | ${ }^{15} \mathrm{~N}$ REDOR SSNMR measurement of labelled RGO. ${ }^{15} \mathrm{~N}$ CPMAS SSNMR spectra of ${ }^{13} \mathrm{C}$ - and ${ }^{15} \mathrm{~N}$-labelled RGO (a) without and (b) with ${ }^{13} \mathrm{C}-{ }^{15} \mathrm{~N}$ dipolar dephasing by (c) a REDOR pulse sequence with a dephasing period of $1.6 \mathrm{~ms}$, (a) without and (b) with ${ }^{15} \mathrm{~N} \pi$-pulse trains for the ${ }^{15} \mathrm{~N}$ channel. Although the ${ }^{15} \mathrm{~N}$-signal intensities for both control and dephased spectra are mildly modulated by ${ }^{15} \mathrm{~N} T_{2}$ relaxation, residual ${ }^{1} \mathrm{H}-$ ${ }^{15} \mathrm{~N}$ dipolar couplings under fast MAS, and ${ }^{15} \mathrm{~N}-{ }^{15} \mathrm{~N}$ J-coupling, the signal of ${ }^{15} \mathrm{~N}$ species within a few bonds from ${ }^{13} \mathrm{C}$ are substantially suppressed by ${ }^{15} \mathrm{~N}-{ }^{13} \mathrm{C}$ dipolar couplings when a ${ }^{13} \mathrm{C} \pi$-pulse train is applied as shown in $\mathbf{b}$. The phase cycles in $\mathbf{c}$ are as follows: $\phi_{1}=x,-x ; \phi_{2}=y, y,-x,-x$. The receiver phase was cycled as $x,-x, y,-y$. The phases for $\phi_{3}$ and $\phi_{4}$ in the rotor-synchronous $\pi$-pulse trains were incremented after every $\pi$-pulse, following the $\mathrm{XY}-16$ phase cycle. $\tau_{\mathrm{R}}$ denotes one rotation period during magic angle spinning.

the short contact time of $0.35 \mathrm{~ms}$ used in the CP period, the signal is likely due to protonated ${ }^{15} \mathrm{~N}$ atoms. From the data, an $\mathrm{N}$ atom ( $-\mathrm{NH} 2$ moiety) in a hydrazone group $\left(-\mathrm{C}=\mathrm{N}-\mathrm{NH}_{2}\right)$ can be ruled out because NMR signals corresponding to $-\mathrm{NH}_{2}$ groups are usually observed between 0 and 100 p.p.m. in ${ }^{15} \mathrm{~N}$ NMR (ref. 30). Figure $3 \mathrm{~b}$ shows the ${ }^{15} \mathrm{~N}$ MAS spectrum with ${ }^{13} \mathrm{C}-{ }^{15} \mathrm{~N}$ dipolar dephasing, acquired using a REDOR pulse sequence (Fig. 3c). It can be seen that the peak at 190 p.p.m. is considerably decreased by ${ }^{13} \mathrm{C}-{ }^{15} \mathrm{~N}$ dephasing, suggesting covalent attachment of the ${ }^{15} \mathrm{~N}$ species on ${ }^{13} \mathrm{C}$ atoms. On the basis of the SSNMR experiments with labelled RGO samples, we believe this suggests the formation of aromatic pyrazole groups at the edges of platelets after hydrazine treatment. Although the defect a
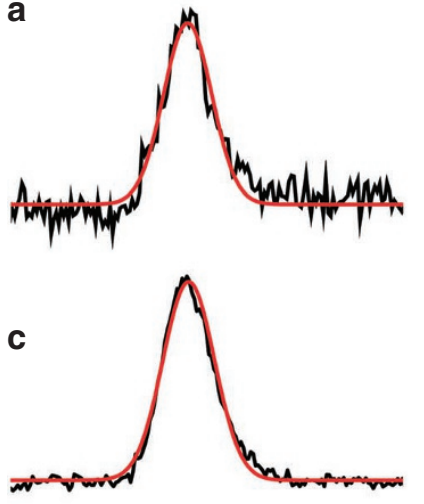

394396398400402404406408 Binding energy (eV)

e

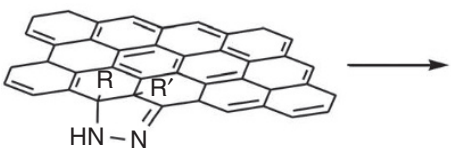

Pyrazoline-like group at the edge

Figure 4 | Chemical structures of unlabelled samples before and after hydrazine treatment. N1s narrow scans in XPS spectra of reduced graphene oxide samples; in $\mathbf{a}$, the reduced graphene oxide is a paperlike material produced by filtration of a suspension of hydrazine-treated graphene oxide platelets; (b), sample (a) following a thermal annealing treatment; (c), a hydrazine vapour-treated graphene oxide film; and (d), sample (c) following a thermal annealing treatment. (e) A schematic diagram for pyrazoline-to-pyrazole conversion at the edges.

structures in the interior of the platelets are not well understood, these pyrazole rings could be generated at the edges around the interior defects.

XPS measurements and calculations on XPS spectra. As the SSNMR spectra of the unlabelled hydrazine-treated graphene oxide showed a limited signal-to-noise ratio and broad signals, XPS was measured on two groups of unlabelled hydrazine-treated graphene oxide samples: graphene oxide platelets in homogeneous colloidal suspensions that were treated with hydrazine and then vacuum filtered to create free-standing 'paper-like' materials ${ }^{35,36}$, and a film composed of graphene oxide platelets spin-coated on glass and then treated with hydrazine vapour. In both cases we observed similar N1s spectra indicating pyrazole moieties, as shown in Figure 4.

The N1s spectra of the 'as-prepared' samples in Figure 4a,c show one symmetrical peak around $400 \mathrm{eV}$, which can be assigned to pyrazoline groups (non-aromatic five-membered rings) rather than pyrazole groups, as discussed below. Although the two $\mathrm{N}$ atoms in pyrazoline groups are not identical, the two N1s peaks in XPS appear at almost the same position, resulting in the generation of one overlapped peak ${ }^{37}$. As shown in Figure 4b,d, thermal annealing (as described in the Methods section) splits the N1s peak into two well-resolved lines at 398.6 and $400.5 \mathrm{eV}$, suggesting that two $\mathrm{N}$ atoms have considerably different chemical environments as observed in pyrazole groups. While the twofold-coordinated $\mathrm{N}$ atom in the pyrazole group has a localized lone pair of electrons, the threefold-coordinated $\mathrm{N}$ associates with delocalized electrons in the aromatic ring. Density-functional theory (DFT) calculations ${ }^{37}$ were performed on pyrazole groups at the edges of graphene platelets for understanding their chemical structure, particularly associated with the experimental XPS peak separation of $1.9 \mathrm{eV}$ (ref. 38). 
The N-N moiety of the pyrazole ring in a graphene nanoribbons with zigzag edges (zGNR) consists of two different $\mathrm{N}$ atoms; one is twofold coordinated with a lone pair of electrons, and the other is threefold coordinated with one hydrogen atom in the $\mathrm{sp}^{2}$ planar configuration, as shown in Supplementary Figure S5. The former is pyridinic-like and the latter is quaternary-like. The two $\mathrm{N}$ atoms should be in different ionization states with different N1s corelevel binding energies as in the pyrazole molecule. Our first-principles DFT calculations give a core-level separation of $1.52 \mathrm{eV}$ for pyrazolated zGNR, consistent with the experimental XPS peak separation of $1.9 \mathrm{eV}$. These values are also very close to 1.59 and $1.8 \mathrm{eV}$ for pyrazole molecules obtained from DFT calculation and XPS measurements, respectively.

Unlike the zGNR model, GO may not be perfectly flat because there is often a considerable amount of residual oxygen after a typical hydrazine treatment. If this is indeed the case, the tertiary $\mathrm{N}$ should undergo out-of-plane relaxation as in an amine group with a lone pair, as shown in Supplementary Figure S5. Then, the core level of the amine-like $\mathrm{N}$ should nearly coincide with that of the pyridinic-like $\mathrm{N}$, at around $400 \mathrm{eV}$. Our calculations for a $\mathrm{zGNR}$ distorted by two extra hydroxyls results in nearly coincident core levels. The core-level separation is only $0.22 \mathrm{eV}$, which can be viewed as a single peak in the XPS measurement. The theoretical core-level separation for pyrazoline molecules is $0.17 \mathrm{eV}$.

Therefore, we can explain the single-to-double transition of N1s XPS peaks on thermal treatment as follows. The hydrazine treatment of GO would incorporate many $\mathrm{N}-\mathrm{N}$ moieties into the RGO sample. Because of incomplete reduction of RGO, the incorporated $\mathrm{N}-\mathrm{N}$ moieties have an $\mathrm{sp}^{3}$ configuration as in pyrazoline groups. After further reduction of RGO by thermal treatment, the $\mathrm{N}-\mathrm{N}$ moieties in $\mathrm{RGO}$ are more akin to $\mathrm{sp}^{2}$ pyrazole groups.

Calculation on reaction pathways and electronic structures. To understand the formation mechanism of the pyrazole groups, we considered several possible reaction routes using graphene nanoribbons with zGNR as shown in equations (1-4). DFT calculations also support the formation of five-membered rings at the edges after hydrazine treatment.

$$
\begin{aligned}
\mathrm{zGNR}(=\mathrm{O},=\mathrm{O}) & +\mathrm{NH}_{2} \mathrm{NH}_{2}+0.5 \mathrm{H}_{2} \rightarrow \mathrm{zGNR}(-\mathrm{NNH}-) \\
& +2 \mathrm{H}_{2} \mathrm{O}, \Delta \mathrm{E}=-1.96 \mathrm{eV}
\end{aligned}
$$

$$
\begin{aligned}
\operatorname{zGNR}(=\mathrm{O},-\mathrm{H}) & +\mathrm{NH}_{2} \mathrm{NH}_{2} \rightarrow \mathrm{zGNR}(-\mathrm{NNH}-) \\
& +\mathrm{H}_{2}+\mathrm{H}_{2} \mathrm{O}, \Delta \mathrm{E}=-0.14 \mathrm{eV}
\end{aligned}
$$

$$
\begin{aligned}
\mathrm{zGNR}(-\mathrm{OH},-\mathrm{OH}) & +\mathrm{NH}_{2} \mathrm{NH}_{2} \rightarrow \mathrm{zGNR}(-\mathrm{NNH}-) \\
+ & 0.5 \mathrm{H}_{2}+2 \mathrm{H}_{2} \mathrm{O}, \Delta \mathrm{E}=-0.67 \mathrm{eV}
\end{aligned}
$$

$$
\begin{aligned}
\operatorname{zGNR}(=\mathrm{O},-\mathrm{H}) & +\mathrm{NH}_{2} \mathrm{NH}_{2} \rightarrow \mathrm{zGNR}\left(=\mathrm{NNH}_{2},-\mathrm{H}\right) \\
& +\mathrm{H}_{2} \mathrm{O}, \Delta \mathrm{E}=0.38 \mathrm{eV}
\end{aligned}
$$

Two zigzag edge sites are functionalized as represented by dashes inside the parentheses. $\Delta \mathrm{E}$ is the reaction energy from the DFT calculations, with a negative reaction energy indicating an exothermic reaction.

When a diketone is presented on the zigzag edge, the formation of a pyrazole group is very likely as the reaction energy is about $-2 \mathrm{eV}$, as shown in equation (1). Other types of edge structures containing ketone or hydroxyl groups can also be converted into pyrazole rings and show non-negligible exothermic reaction energies, as shown in equations (2) and (3). On the contrary, the formation of a hydrazone group is not energetically favourable with an endothermic reaction energy of $0.38 \mathrm{eV}$, as shown in equation (4). This is consistent with our experimental observations. It is possible that hydrazine-assisted chemical reduction produced $\mathrm{N}$ atom-free species at the edges, which, in part, might contribute to the disappearance of the NMR peak at 190 p.p.m. that was previously assigned to $\mathrm{C}=\mathrm{O}$ groups at the platelet edges. However, this mechanism is not sufficient to explain the presence of pyrazole/pyrazoline groups as suggested by our SSNMR and XPS data.

In the field of graphene-based materials, the term 'reduction' typically refers to the removal of oxygen atoms from graphene oxide/GO. However, pyrazole formation at the edges is more properly referred to as a 'substitution' rather than a 'reduction' (Fig. 1c). The aromaticity of the pyrazole moiety should enhance electron conduction because of the reduced band gap and the delocalized electronic states associated with the $\mathrm{N}$ in the $\mathrm{sp}^{2}$ configuration. Figure 5 illustrates the total and local densities of states of zGNR with pyrazole groups at the edges, and several electronic states that are associated with the $\mathrm{N}_{2}$ pyrazole moieties. We can clearly identify the localized lone pair electronic state at $-3 \mathrm{eV}$ (below the Fermi level), which corresponds to the pyridinic $\mathrm{N}\left(\mathrm{N}_{\mathrm{P}}\right)$. The electronic state of the $\mathrm{sp}^{2}$ or quaternary $\mathrm{N}\left(\mathrm{N}_{\mathrm{Q}}\right)$ can be found at several energies, for example, $-7.5,-2.4$ and $-2.0 \mathrm{eV}$. The state at $-7.5 \mathrm{eV}$ is pentagonal, as in the aromatic pyrazole. The $\mathrm{N}_{\mathrm{Q}}$ electronic states near the Fermi level are well hybridized with the graphene bulk states. Consequently, aromatic $\mathrm{N}_{2}$ doping (particularly, $\mathrm{N}_{\mathrm{Q}}$ ) strongly influences the electronic structures of hydrazine-treated graphene oxide materials.

\section{Discussion}

The SSNMR measurements revealed the presence of other chemical groups on the labelled RGO sample. A peak corresponding to $\mathrm{sp}^{2}$ bound ${ }^{13} \mathrm{C}$ atoms in $\mathrm{GO}$ at roughly 130 p.p.m. had shifted upfield to 118 p.p.m. as shown in Figure 1b. This is consistent with previous reports on the SSNMR spectra of unlabelled, reduced graphene oxides $^{3,22}$. The peak shift indicates a greater amount of $\mathrm{sp}^{2}$ conjugation in RGO than in GO, thus suggesting that the $\mathrm{sp}^{2}$ network was restored by hydrazine treatment. A peak at approximately $70 \mathrm{p}$. p.m. corresponding to hydroxyl $(-\mathrm{COH})$ groups $^{18,19}$ was nearly removed, while a peak at 60 p.p.m., previously assigned to 1,2-epoxide functionalities ${ }^{18,19}$, was completely suppressed after hydrazine treatment. This suggests that hydroxyl groups on the basal plane were mostly removed and 1,2-epoxide groups on the basal planes were completely removed by the hydrazine treatment (within the detection limit of these NMR measurements). In other words, the basal planes of GO have largely been reduced by the hydrazine treatment. Although the mechanism is not well understood, the reduction of GO is generally believed to restore the $\mathrm{sp}^{2}$ networks on the basal planes while reducing the concentration of hydroxyl and 1,2-epoxide groups.

The region around 170 p.p.m. in Figure $1 \mathrm{~b}$, which was previously assigned to carboxyl groups $(-\mathrm{COOH})$ at the edges of platelets of $\mathrm{RGO}^{18,19}$, overlaps with a tail of the peak at 150 p.p.m. Consequently, it is difficult to conclusively determine whether carboxyl groups remain in the RGO sample based on the peak assigned to them. It is well known that carboxyl groups can be reduced to hydroxyl groups by reaction with strong reductants such as lithium aluminium hydride ${ }^{32,33}$. However, in the case of hydrazine treatment under the reaction conditions used here, the reduction of carboxyl groups to hydroxyl groups could be ruled out due to the absence of a peak corresponding to hydroxyl groups in the ${ }^{13} \mathrm{C}$ NMR spectrum of labelled RGO. In addition, it may be expected that the carboxyl groups can be converted to amide groups $\left(-\mathrm{CO}-\mathrm{NH}-\mathrm{NH}_{2}\right)$ by reaction with hydrazine. However, the ${ }^{15} \mathrm{~N}$ SSNMR spectra did not indicate the presence of $-\mathrm{NH}_{2}$ groups, thus suggesting that no amide groups were generated.

In conclusion, we investigated the chemical structures of hydrazine-treated graphene oxide/GO, which is one of the most widely 


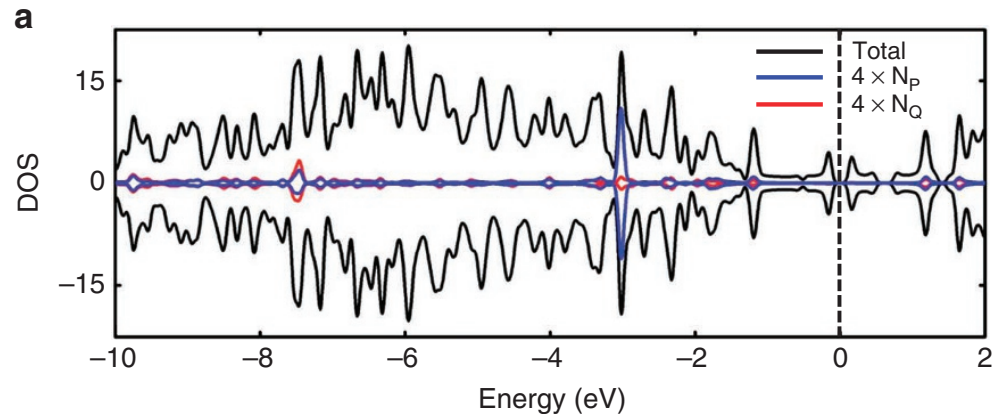

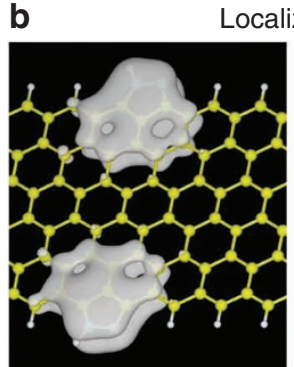

$-7.46 \mathrm{eV}$
Localized state

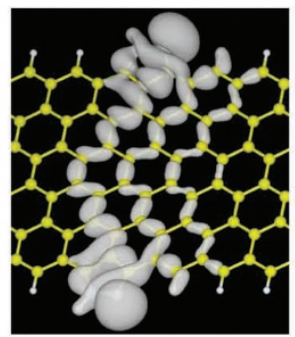

$-3.02 \mathrm{eV}$
C

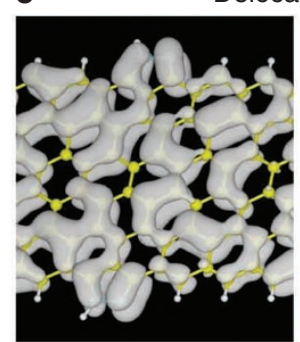

$-2.40 \mathrm{eV}$
Delocalized state

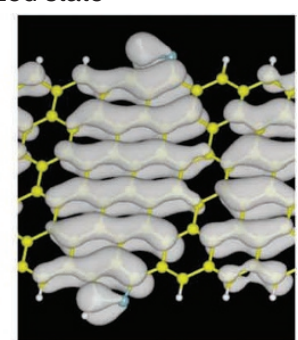

$-1.98 \mathrm{eV}$

Figure $\mathbf{5}$ | Theoretical calculation on electronic structures of RGO materials with $\mathbf{N}_{\mathbf{2}}$ doping at the edges. (a) Density of states (DOS) of a zigzag graphene nanoribbon with pyrazoles at both the edges, and $(\mathbf{b}, \mathbf{c})$ a set of charge density plots of $\mathrm{N}_{2}$-related electronic states at several energies for (b) localized states and (c) delocalized states.

used graphene-based materials, using SSNMR spectroscopy and XPS. We first produced ${ }^{13} \mathrm{C}$ - and ${ }^{15} \mathrm{~N}$-labelled RGO materials by reaction of ${ }^{13} \mathrm{C}$-labelled $\mathrm{GO}$ with ${ }^{15} \mathrm{~N}$-labelled hydrazine monohydrate. The isotope-labelled materials showed tremendously improved SSNMR signals relative to unlabelled material and we found that the hydrazine treatment caused the formation of pyrazole groups-aromatic five-membered rings with two adjacent $\mathrm{N}$ atoms - at the edges of resulting platelets. Furthermore, XPS experiments on unlabelled, hydrazine-treated graphene oxide revealed the formation of five-membered pyrazoline or pyrazole rings (after heat treatment) at the edges. The evidence of chemical substitution by hydrazine may offer a novel path to selective chemical modifications at the edges of graphene platelets for graphene-based systems. For example, an acidic proton in the pyrazole rings could be activated by treatment with a strong base for further modification ${ }^{31}$.

The methods and results presented here show that isotopic labelling with NMR-active nuclei such as ${ }^{13} \mathrm{C}$ and ${ }^{15} \mathrm{~N}$, particularly when combined with other spectroscopic methods such as XPS, can provide detailed insight into the chemical structure of CMGs. The SSNMR spectra of ${ }^{13} \mathrm{C}$ - and ${ }^{15} \mathrm{~N}$-labelled RGO also showed almost complete removal of epoxy and hydroxyl groups, and significant restoration of the $\mathrm{sp}^{2}$ network on the basal planes. On the basis of our analysis, we suggested that the term 'hydrazine-reduced/substituted graphene oxide' is more correct and descriptive than 'hydrazinereduced graphene oxide' which, to date, has generally been used in the peer-reviewed literature to refer to this material.

\section{Methods}

Preparation of ${ }^{13} \mathrm{C}$-labelled graphite. Uniformly ${ }^{13} \mathrm{C}$-labelled graphite films were prepared using a cold-wall CVD system, as described previously ${ }^{18}$. The graphite was obtained by precipitation from the Ni foil during cooling, and formed on both sides of the Ni foil. The ${ }^{13} \mathrm{C}$-labelled graphite film was removed from the $\mathrm{Ni}$ substrate using scotch tape. The graphite films on the scotch tape were immersed in acetone for 1 day to dissolve the tape, then sonicated for $30 \mathrm{~min}$. The supernatant was decanted and this dissolving and decanting process was repeated two more times. After filtration followed by washing with dimethylformamide, ethanol and acetone, three times each, the product was dried in the oven $\left(60^{\circ} \mathrm{C}\right)$ for 2 days, and ${ }^{13} \mathrm{C}$-labelled graphite flake was obtained.
Preparation of ${ }^{13} \mathrm{C}$ - and ${ }^{15} \mathrm{~N}$-labelled RGO. ${ }^{13} \mathrm{C}$-labelled GO was produced by the modified Hummers method ${ }^{39}$. Control experiments were used to determine the proper amounts of hydrazine monohydrate to add for the creation of RGO samples. We tested the effect of the amount of hydrazine monohydrate relative to GO in liquid-phase reaction without isotope labelling. It was found that $1 \mu \mathrm{l}$ of hydrazine monohydrate is sufficient to reduce $3 \mathrm{mg}$ of GO (data not shown). Consequently, we used the same ratio of hydrazine monohydrate to GO with the labelled chemicals in this work.

The ${ }^{13} \mathrm{C}$-labelled graphite $(60 \mathrm{mg})$ was added to a flask filled with $\mathrm{H}_{2} \mathrm{SO}_{4}$ $(30 \mathrm{ml})$, then the mixture was sonicated for $2 \mathrm{~h}$. Solid $\mathrm{KMnO}_{4}(300 \mathrm{mg}, 99.2 \%$, Fisher Scientific) was then slowly added to the flask in an ice bath. After addition of the $\mathrm{KMnO}_{4}$, the flask was immersed in a water bath at $35^{\circ} \mathrm{C}$, and stirred with a magnetic bar for $24 \mathrm{~h}$. Deionized water $(150 \mathrm{ml})$ was added to the flask in the ice bath, followed by stirring in this ice bath for $1 \mathrm{~h}$. The flask was then taken off the ice bath and stirred at room temperature for $2 \mathrm{~h}$. Hydrogen peroxide (30 wt $\%$ in water, Sigma-Aldrich) was then added to the mixture until no further gas evolution was observed, followed by stirring for $2 \mathrm{~h}$. The mixture was then filtered using an Anodisc membrane filter ( $47 \mathrm{~mm}$ in diameter, $0.2-\mu \mathrm{m}$ pore size, Whatman, Middlesex, UK), followed by air drying for 1 week. Then, two batches of air-dried, ${ }^{13} \mathrm{C}$-labelled GO were placed in a vial with water $(15 \mathrm{ml})$, followed by addition of ${ }^{15} \mathrm{~N}$-labelled hydrazine monohydrate ( 98 atom $\%{ }^{15} \mathrm{~N}$, Sigma-Aldrich; $1 \mu \mathrm{l}$ for $3 \mathrm{mg}$ of GO). The mixture was stirred at $80^{\circ} \mathrm{C}$ for 1 day. After cooling to room temperature, the mixture was filtered using the Anodisc membrane filter, followed by air drying for 1 day, and finally dried under vacuum for 1 day. One batch of the sample (roughly $2 \mathrm{mg}$ ) was used to acquire the data as in Figures $1 \mathrm{~b}, 2$. The second batch $(10 \mathrm{mg})$, which had a lower degree of reduction, was used to acquire the data as in Figure 3 and Supplementary Figure S3.

Heat treatment of graphene oxide in water without hydrazine. An aqueous suspension of unlabelled graphene oxide ( $3 \mathrm{mg}$ of GO per $1 \mathrm{ml}$ of purified water) was prepared by sonication of GO, prepared via the modified Hummers method and derived from natural graphite (SP-1, Bay Carbon) as described previously. This suspension was stirred at $80^{\circ} \mathrm{C}$ for $12 \mathrm{~h}$ without hydrazine. The suspension was stable and the colour of the suspension was unchanged by the treatment. A paperlike material was prepared by filtration of the resulting suspension as previously reported $^{35,36}$, and this material was used for XPS measurements.

SSNMR measurements. SSNMR experiments were conducted at $9.4 \mathrm{~T}$ using a Varian InfinityPlus 400 NMR spectrometer and a home-built 2.5-mm tripleresonance MAS probe at a ${ }^{1} \mathrm{H}$ NMR frequency of $400.214 \mathrm{MHz}$. All ${ }^{13} \mathrm{C}$ chemical shifts were referenced to tetramethylsilane using adamantane as an external reference. The ${ }^{15} \mathrm{~N}$ chemical shifts were referenced to liquid ${ }^{15} \mathrm{NH}_{3}$ at 0 p.p.m. using the indirect referencing of a ${ }^{13} \mathrm{C}$ signal for adamantane ${ }^{40,41}$. In all the experiments, the external air temperature was set to $-10^{\circ} \mathrm{C}$ using a Varian VT controller, and the MAS spinning speed was $20,000 \pm 10 \mathrm{~Hz}$. 
For the measurement shown in Figure $1 \mathrm{~b}, \mathrm{a}{ }^{13} \mathrm{C}$ single-pulse echo sequence was used with a $\pi / 2$ pulse of $2.5-\mu$ s width and no ${ }^{1} \mathrm{H}$ decoupling. We confirmed that removing ${ }^{1} \mathrm{H}$ decoupling did not alter the resolution. A separate ${ }^{13} \mathrm{C}-{ }^{1} \mathrm{H}$ REDOR experiment for the RGO sample confirmed that the peaks at 120 and 150 p.p.m. in Figure $1 \mathrm{~b}$ could likely be associated with non-protonated carbons. Thus, fast spinning at $20 \mathrm{kHz}$ sufficiently removed line broadening due to ${ }^{1} \mathrm{H}-{ }^{13} \mathrm{C}$ dipolar couplings.

For Figure 2a,b, a total of 5,400 scans were collected with recycle delays of $5 \mathrm{~s}$ and ${ }^{15} \mathrm{~N}$ and ${ }^{13} \mathrm{C} \pi$-pulse widths of 15 and $10 \mu$ s, respectively. $\mathrm{A}^{13} \mathrm{C}-{ }^{15} \mathrm{~N}$ REDOR pulse sequence (Fig. $2 \mathrm{~d}$ ) was used with a total of 32 rotor periods $(1.6 \mathrm{~ms})$ for dipolar dephasing. ${ }^{1} \mathrm{H}$ decoupling during the REDOR period was $55 \mathrm{kHz}$; we confirmed that there was little change in the spectra without ${ }^{1} \mathrm{H}$ decoupling. No ${ }^{1} \mathrm{H}$ decoupling was applied during the acquisition period. The spinning speed of $20,000 \pm 10 \mathrm{~Hz}$ was used to remove relatively strong ${ }^{13} \mathrm{C}-{ }^{13} \mathrm{C}$ dipolar couplings. The $\pi$-pulse widths were 10 and $15 \mu$ s for ${ }^{13} \mathrm{C}$ and ${ }^{15} \mathrm{~N}$, respectively. In these REDOR experiments, the ${ }^{13} \mathrm{C}$ nuclei in the vicinity of ${ }^{15} \mathrm{~N}$ nuclei lose their signals in the ${ }^{13} \mathrm{C}$ MAS spectrum with ${ }^{13} \mathrm{C}-{ }^{15} \mathrm{~N}$ dipolar dephasing. In a control REDOR experiment for uniformly ${ }^{13} \mathrm{C}$ - and ${ }^{15} \mathrm{~N}$-labelled L-alanine ${ }^{24}$, a signal for ${ }^{13} \mathrm{C}_{\alpha}$ that is directly bonded to ${ }^{15} \mathrm{NH}_{3}{ }^{+}$decreased by $78 \%$, compared with the control spectrum without ${ }^{13} \mathrm{C}-{ }^{15} \mathrm{~N}$ dipolar dephasing. In the same study, signals for $-\mathrm{CO}_{2}{ }^{-}$and $-\mathrm{CH}_{3}$, which are two bonds away from ${ }^{15} \mathrm{~N}$, decreased by only 6 and $8 \%$, respectively. The integrated intensity of the difference spectrum in Figure $2 \mathrm{c}$ is about $7.5 \pm 0.5 \%$ with respect to that of Figure 2a. Although the signal intensities in the experiment are modulated by ${ }^{13} \mathrm{C}-{ }^{13} \mathrm{C}$ J-coupling and ${ }^{13} \mathrm{C}$ transverse spin relaxation, the result indicates that a considerable fraction of ${ }^{13} \mathrm{C}$ atoms observed here are likely to be bonded to ${ }^{15} \mathrm{~N}$ species. Gaussian line broadening of $200 \mathrm{~Hz}$ was applied.

For Figure 3a,b, a pulse sequence in Figure $3 \mathrm{c}$ was used. First, ${ }^{15} \mathrm{~N}$ spin polarization was prepared by ramped $\mathrm{CP}^{42}$. Because of the limited population of the target ${ }^{15} \mathrm{~N}$ species and a broad line width, the signal-to-noise ratio in the direct-detected ${ }^{15} \mathrm{~N}$ CPMAS spectra was low even with ${ }^{15} \mathrm{~N}$ enrichment. Thus, we used ${ }^{1} \mathrm{H}$ detected ${ }^{15} \mathrm{~N}-{ }^{1} \mathrm{H}$ double $\mathrm{CP}$ for the adjustment of the Hartmann-Hahn condition ${ }^{43,44}$. The ${ }^{1} \mathrm{H}$ polarization was excited by a ${ }^{1} \mathrm{H} \pi / 2$ pulse of $3.5-\mu$ s width. During a contact period of $0.35 \mathrm{~ms}$ for polarization transfer by $\mathrm{CP}$, the ${ }^{15} \mathrm{~N}$ radiofrequency (RF) field amplitude was linearly swept from 21 to $36 \mathrm{kHz}$, while the ${ }^{1} \mathrm{H}$ RF amplitude was kept constant at $48 \mathrm{kHz} .{ }^{15} \mathrm{~N}$ and ${ }^{13} \mathrm{C} \pi$-pulse widths in the REDOR sequence were $15 \mu \mathrm{s}$. The signals of 21,504 scans were accumulated with recycle delays of $3 \mathrm{~s}$. Gaussian line broadening of $400 \mathrm{~Hz}$ was applied. The total experimental time was $\sim 19 \mathrm{~h}$ for each sample.

XPS measurements. An aqueous suspension of graphene oxide was prepared by exfoliation of GO. The aqueous suspension $\left(3.1 \mathrm{mg} \mathrm{ml}^{-1}\right)$ was diluted with ethano for facile spin casting onto glass substrates (Corning 1737). Graphene oxide thin films were prepared by spin casting the solution on a glass substrate cleaned by ultraviolet oxygen radiation. The films were subsequently reduced by a 1 -h exposure to hydrazine vapour generated at $100^{\circ} \mathrm{C}$ in a sealed container. One subset of the hydrazine vapour-treated samples was set aside for testing after the hydrazine vapour treatment. The remaining vapour-treated samples were reduced with thermal annealing at $750^{\circ} \mathrm{C}$ in a CVD chamber under $\mathrm{H}_{2}$ flow $(100 \mathrm{sccm})$ for $5 \mathrm{~min}$. Paper-like materials of reduced graphene oxide were prepared by simple filtration as previously reported ${ }^{35,36}$. Thermal annealing of the paper sample was done under Ar flow for $12 \mathrm{~h}$ at $500^{\circ} \mathrm{C}$. After the reduction process, surface chemical analysis of each reduced graphene oxide sample was performed by using XPS (Sigma Probe, Thermo VG Scientific).

DFT calculations on the pyrazole formation. For DFT calculations, we used the projector-augmented wave potentials ${ }^{45}$ with a plane-wave basis set and the Perdew-Burke-Ernzernhof ${ }^{46}$ exchange-correlation functional as implanted in the Vienna Ab-Initio Simulation Package ${ }^{38}$. A kinetic energy cutoff of $400 \mathrm{eV}$ and Gaussian smearing of $0.05 \mathrm{eV}$ was used. A (6×6) zigzag graphene nanoribbon supercell with vacuum separation of $10 \AA$ and a $(8 \times 1 \times 1) \mathbf{k}$-points sampling were used in calculations. All atomic forces were minimized at $<0.025 \mathrm{eV}^{-1}$, and the in-plane stresses were fully relaxed.

\section{References}

1. Dobelle, W. H. \& Beer, M. Chemically cleaved graphite support films for electron microscopy. J. Cell Biol. 39, 733-735 (1968).

2. Boehm, V. H. P., Clauss, A., Fischer, G. O. \& Hofmann, U. Dunnste Kohlenstoff-folien. Z. Naturforschg. 17b, 150-153 (1962).

3. Stankovich, S. et al. Synthesis of graphene-based nanosheets via chemical reduction of exfoliated graphite oxide. Carbon 45, 1558-1565 (2007).

4. Si, Y. \& Samulski, E. T. Synthesis of water soluble graphene. Nano Lett. 8, 1679-1682 (2008)

5. Wang, G. et al. Facile synthesis and characterization of graphene nanosheets. J. Phys. Chem. C 112, 8192-8195 (2008).

6. Park, S. et al. Colloidal suspensions of highly reduced graphene oxide in a wide variety of organic solvents. Nano Lett. 9, 1593-1597 (2009).

7. Watcharotone, S. et al. Graphene-silica composite thin films as transparent conductors. Nano Lett. 7, 1888-1892 (2007).
8. Park, S. \& Ruoff, R. S. Chemical methods for the production of graphenes. Nat. Nanotechnol. 4, 217-224 (2009).

9. Loh, K. P., Bao, Q., Eda, G. \& Chhowalla, M. Graphene oxide as a chemically tunable platform for optical applications. Nat. Chem. 2, 1015-1024 (2010).

10. Zhu, Y. et al. Graphene and graphene oxide: synthesis, properties, and applications. Adv. Mater. 22, 3906-3924 (2010).

11. Dreyer, D. R., Park, S., Bielawski, C. W. \& Ruoff, R. S. The chemistry of graphene oxide. Chem. Soc. Rev. 39, 228-240 (2010).

12. Lee, D. H. et al. Versatile carbon hybrid films composed of vertical carbon nanotubes grown on mechanically compliant graphene films. Adv. Mater. 22, $1247-1252$ (2010).

13. Potts, J. R., Dreyer, D. R., Bielawski, C. W. \& Ruoff, R. S. Graphene-based polymer nanocomposites. Polymer 52, 1-25 (2011).

14. Hwang, J. O. et al. Workfunction-tunable, $\mathrm{N}$-doped reduced graphene transparent electrodes for high-performance polymer light-emitting diodes. ACS Nano (in press), doi:10.1021/nn203176u.

15. Wang, X. et al. N-doping of graphene through electrothermal reactions with ammonia. Science 324, 768-771 (2009).

16. Lerf, A., He, H., Riedl, T. \& Klinowski, J. ${ }^{13} \mathrm{C}$ and ${ }^{1} \mathrm{H}$ MAS NMR studies of graphite oxide and its chemically modified derivatives. Solid State Ionics 101-103, 857-862 (1997).

17. He, H., Riedl, T., Lerf, A. \& Klinowski, J. Solid-state NMR studies of the structure of graphite oxide. J. Phys. Chem. 100, 19954-19958 (1996).

18. Cai, W. et al. Synthesis and solid-state NMR structural characterization of ${ }^{13} \mathrm{C}$-labeled graphite oxide. Science 321, 1815-1817 (2008)

19. Casabianca, L. B. et al. NMR-based structural modeling of graphite oxide using multidimensional ${ }^{13} \mathrm{C}$ solid-state NMR and ab initio chemical shift calculation. J. Am. Chem. Soc. 132, 5672-5676 (2010).

20. He, H., Klinowski, J., Forster, M. \& Lerf, A. A new structural model for graphite oxide. Chem. Phys. Lett. 287, 53-56 (1998).

21. Rodriguez, A. M. \& Jimenez, P. S. V. Some new aspects of graphite oxidation at $0^{\circ} \mathrm{C}$ in a liquid medium. A mechanism proposal for oxidation to graphite oxide. Carbon 24, 163-167 (1986).

22. Gao, W., Alemany, L. B., Ci, L. \& Ajayan, P. M. New insights into the structure and reduction of graphite oxide. Nat. Chem. 1, 403-408 (2009).

23. Gullion, T. \& Schaefer, J. Detection of weak heteronuclear dipolar coupling by rotational-echo double-resonance. Adv. Magn. Reson. 13, 57-83 (1989).

24. Marshall, G. R. et al. Determination of a precise interatomic distance in a helical peptide by REDOR NMR. J. Am. Chem. Soc. 112, 963-966 (1990).

25. Kondo, T., Honma, Y., Oh, J., Machida, T. \& Nakamura, J. Edge states propagating from a defect of graphite: scanning tunneling spectroscopy measurements. Phys. Rev. B 82, 153414 (2010).

26. Bagri, A. et al. Structural evolution during the reduction of chemically derived graphene oxide. Nat. Chem. 2, 581-587 (2010).

27. Griffin, R. G. Dipolar recoupling in MAS spectra of biological solids. Nat. Struct. Mol. Biol. 5, 508-512 (1998).

28. McDermott, A. E. Structural and dynamic studies of proteins by solid-state NMR spectroscopy: rapid movement forward. Curr. Opin. Struct. Biol. 14, 554-561 (2004)

29. Chimon, S. et al. Evidence of fibril-like $\beta$-sheet structures in neurotoxic amyloid intermediate for Alzheimer's $\beta$-amyloid. Nat. Struct. Mol. Biol. 14, 1157-1164 (2007).

30. Levy, G. C. \& Lichter, R. L. Nitrogen-15 Nuclear Magnetic Resonance Spectroscopy (John Wiely \& Sons Inc., 1979).

31. Joule, J. A., Mills, K. \& Smith, G. F. Heterocyclic Chemistry (Chapman \& Hall, 1995).

32. McMurry, J. E. Organic Chemistry (Thomson, Brooks/Cole, 2003).

33. Morrison, R. T. \& Boyd, R. N. Organic Chemistry (Prentice-Hall Inc., 1992).

34. Aguilarparrilla, F., Mannle, F., Limbach, H. H., Elguero, J. \& Jagerovic, N. N-15 NMR chemical-shifts of NH-pyrazoles in the solid-state and in solution at lowtemperature. Magn. Reson. Chem. 32, 699-702 (1994).

35. Dikin, D. A. et al. Preparation and characterization of graphene oxide paper. Nature 448, 457-460 (2007).

36. Park, S. et al. Graphene oxide papers modified by divalent ions-enhancing mechanical properties via chemical cross-linking. ACS Nano 2, 572-578 (2008).

37. Katrib, A., El-Rayyes, N. R. \& Al-Kharafi, F. M. N 1s orbital binding energies of some pyrazole and pyrazoline compounds by XPS. J. Electron Spectrosc. 31 317-321 (1983)

38. Kresse, G. \& Joubert, D. From ultrasoft pseudopotentials to the projector augmented-wave method. Phys. Rev. B 59, 1758-1775 (1999).

39. Park, S. et al. Aqueous suspension and characterization of chemically modified graphene sheets. Chem. Mater. 20, 6592-6594 (2008).

40. Morcombe, C. R. \& Zilm, K. W. Chemical shift referencing in MAS solid state NMR. J. Magn. Reson. 162, 479-486 (2003).

41. Wishart, D. S. et al. ${ }^{1} \mathrm{H},{ }^{13} \mathrm{C}$ and ${ }^{15} \mathrm{~N}$ chemical shift referencing in biomolecular NMR. J. Biomol. NMR 6, 135-140 (1995).

42. Metz, G., Wu, X. \& Smith, S. O. Ramped-amplitude cross polarization in magic-angle-spinning NMR. J. Magn. Reson. Ser. A 110, 219-227 (1994). 
43. Ishii, Y. \& Tycko, R. Sensitivity enhancement in solid state ${ }^{15} \mathrm{~N}$ NMR by indirect detection with high-speed magic angle spinning. J. Magn. Reson. 142, 199-204 (2000).

44. Ishii, Y., Yesinowski, J. P. \& Tycko, R. Sensitivity enhancement in solid-state C-13 NMR of synthetic polymers and biopolymers by H-1 NMR detection with high-speed magic angle spinning. J. Am. Chem. Soc. 123, 2921-2922 (2001).

45. Blochl, P. E. Projector augmented-wave method. Phys. Rev. B 50, 17953 (1994).

46. Perdew, J. P., Burke, K. \& Ernzerhof, M. Generalized gradient approximation made simple. Phys. Rev. Lett. 77, 3865-3868 (1996).

\section{Acknowledgements}

S.P. and J.O. were supported by Global Frontier Research Center for Advanced Soft Electronics. This study was supported in part by the U.S. Department of Energy, Office of Basic Energy Sciences, Division of Materials Sciences and Engineering under Award DESC001951 (R.S.R and Y.I.). The SSNMR methodology development in this work was also supported by the NSF (CHE 449952, CHE 957793) and the Dreyfus Foundation TeacherScholar Award program for Y.I. L.B.C. held an American Fellowship from the American Association of University Women during the course of this work. J.O.H and S.O.K. were supported by the NRL (R0A-2008-000-20057-0) and WCU (R32-2008-000-10051-0) programs funded by the Korean government. E.-S.L and Y.-H.K were supported by the
WCU (R31-2008-000-10071-0) and Basic Science Research (2010-0006922) programs through the NRF of Korea.

\section{Author contributions}

S.P. organized experiments, synthesized materials and wrote the manuscript. Y.H. and L.B.C. measured NMR spectra and J.O.H. measured XPS spectra. E.-S.L. contributed theoretical calculations. W.C., S.C., H.-W.H. and J.O. assisted with the synthesis of materials. J.R.P. conducted data analysis. S.O.K. supervised XPS measurements and gave intellectual input. Y.-H.K. supervised XPS measurements and theoretical calculations and wrote the manuscript. Y.I. performed and supervised NMR measurements, analysed the NMR data for the structural analysis and wrote the manuscript. R.S.R. supervised synthesis of materials and wrote the manuscript.

\section{Additional information}

Supplementary Information accompanies this paper at http://www.nature.com/ naturecommunications

Competing financial interests: The authors declare no competing financial interests.

Reprints and permission information is available online at http://npg.nature.com/ reprintsandpermissions/

How to cite this article: Park, S. et al. Chemical structures of hydrazine-treated graphene oxide and generation of aromatic nitrogen doping. Nat. Commun. 3:638 doi: $10.1038 /$ ncomms1643 (2012). 